Tohoku J. Exp. Med., 2008, 215, 89-93

\title{
Portal Vein Hemodynamics in Patients with Non-Alcoholic Fatty Liver Disease
}

\author{
Besir Erdogmus, ${ }^{1}$ Ali Tamer, ${ }^{2}$ Ramazan Buyukkaya,,${ }^{1}$ Burhan Yazici, ${ }^{1}$ \\ Ayla Buyukkaya, ${ }^{1}$ Esin Korkut, ${ }^{3}$ Aytekin Alcelik ${ }^{3}$ and Ugur Korkmaz ${ }^{3}$ \\ ${ }^{1}$ Department of Radiology, Duzce University, Medical School, Duzce, Turkey \\ ${ }^{2}$ Department of Internal Medicine, Abant Izzet Baysal University, Medical School, Duzce, Turkey \\ ${ }^{3}$ Department of Internal Medicine, Duzce University, Medical School, Duzce, Turkey
}

\begin{abstract}
Paralleling the rise in the incidence of obesity and diabetes worldwide, nonalcoholic fatty liver disease (NAFLD) is being increasingly recognized as one of the major causes of chronic liver disease. Doppler sonography is used as a diagnostic method in the non-invasive assessment of the hemodynamics of hepatic vascular flow in liver diseases. We investigated the effects of fatty infiltration in the liver on the Doppler flow hemodynamics of the portal vein. Doppler sonography of the liver and portal vein was performed in 60 subjects with NAFLD and 20 healthy volunteers (control). The patients were grouped into mild (grade 1), moderate (grade 2), and severe (grade 3) according to sonographic appearance of hepatosteatosis ( $n=20$ for each group). The vein pulsatility index (VPI), mean flow velocity $(\mathrm{MFV})$, peak maximum velocity $\left(\mathrm{V}_{\max }\right)$, and peak minimum velocity $\left(\mathrm{V}_{\min }\right)$ of the portal vein were significantly lower in patients with NAFLD than those of the controls $(p<0.001)$. The VPI was 0.20 in the patients and 0.31 in the control. The MFV was 12.3 $\mathrm{cm} / \mathrm{sec}$ in the patients and $16.5 \mathrm{~cm} / \mathrm{sec}$ in the control group. The portal vein flow was found to be decreased as the grade of fatty infiltration increased for VPI ( $\mathrm{r}=-0.946, p<$ $0.001)$, MFV $(\mathrm{r}=-0.951, p<0.001)$. The alteration in Doppler waveform pattern of portal vein with fatty liver population suggests reduced vascular compliance in the liver. doppler sonography; nonalcoholic fatty liver; portal vein pulsatility; hepatosteatosis; liver. Tohoku J. Exp. Med., 2008, 215 (1), 89-93.
\end{abstract}

(C) 2008 Tohoku University Medical Press

Fatty liver is a relatively common incidental finding on imaging studies. Although generally a benign condition, fat in the liver can be troubling for clinicians because it can cause persistently elevated liver enzyme levels (Neuschwander. 2001). Imaging studies assist in the diagnosis of nonalcoholic fatty liver disease (NAFLD) through identifying fatty infiltrate in the liver. Ultrasonography of the liver has a sensitivity of 82 to $89 \%$ and a specificity of $93 \%$ for identifying fatty liver infiltrate (Bayard et al. 2006). Paralleling the rise in the incidence of obesity and diabetes worldwide, NAFLD is being increasingly recognized as one of the major causes of chronic liver disease (Flegal et al. 1998; Salgado et al. 2006). Steatohepatitis occurs in 24 to $95 \%$ of these patients, whereas fibrosis is implicated in 6 to $74 \%$ of the cases, and cirrhosis in 0 to $24 \%$ (Kral et al. 2004; Salgado et al. 2006).

Doppler sonography is used as a major diag-

Received February 20, 2008; revision accepted for publication April 2, 2008.

Correspondence: Besir Erdogmus, Duzce University, Duzce Medical Faculty, Department of Radiology, 81620 Konuralp, Duzce, Turkey.

e-mail: besirerdogmus@yahoo.com 
nostic method in the non-invasive assessment of the hemodynamics of hepatic vascular flow (Aube et al. 2004). Previous studies have shown that the flow of the hepatic and portal veins is altered in patients with liver cirrhosis, portal vein thrombosis, Budd-Chiari syndrome, and vascular malformations (Dietrich et al. 1998; Bargallo et al. 2006; Bolognesi et al. 2007). However, a recent study with a similar design has been published showing the relationship between fatty infiltration of the liver and decreased portal venous velocity and pulsatility (Balci et al. 2008). We also aimed to investigate the effect of fatty infiltration on the Doppler flow volume of the portal vein.

\section{Materials And Methods}

Ultrasonographic examination of the portal vein was performed on 80 subjects ( 60 patients with fatty livers; 32 males; 28 females, with a mean age of 42.96 years [range 18-69 years] and 20 healthy volunteers; 10 males; 10 females, with a mean age of 44.50 years [range 30-67 years]) who were consecutively admitted to gastroenterology outpatient clinic. Three groups of 20 patients each with different degrees of fatty infiltration of the liver were selected. The groups were classified as the control (grade 0 ), mild (grade 1), moderate (grade 2), and severe (grade 3 ) groups according to sonographic appearance of hepatosteatosis. Subjects with heart disease based on medical history, physical examination, and chest radiography were excluded. Patients with acute or chronic liver disorders and malignancy were also eliminated based on appropriate clinical, laboratory, and imaging investigations. All the subjects in the study and control groups were negative for hepatitis B surface antigen and anti-hepatitis $\mathrm{C}$ virus antibody. To preclude possible influences on the waveform of the vascular hemodynamics, pregnant subjects, patients with a history of abdominal or thoracic surgery, and patients consuming more than $40 \mathrm{~g}$ of alcohol per week or taking cardiovascular drugs or other medications that might have an adverse effect on the liver were excluded from the study and control groups (Karabulut et al. 2004). The procedure and purpose of the examination were explained to all subjects prior to obtaining informed consent.

B-mode and duplex Doppler sonographic examinations were performed by the same radiologist using the same duplex Doppler system (EUB 6500; Hitachi, Tokyo) with a $3.5-\mathrm{MHz}$ convex probe. The radiologist is not blinded when obtaining and grading portal venous waveforms. After an overnight fast, each subject was examined in the supine or $50-60^{\circ}$ left lateral position with the arms raised above the head. The liver parenchyma was scanned and vascular malformations and spaceoccupying lesions were excluded. Using B-mode sonography, the presence or absence and severity of fatty infiltration was graded using a scale from 0 to 3 as previously reported by Mihmanli et al. (2005), indicating absent, mild, moderate, and severe hepatosteatosis, respectively, corresponding to increasing degrees of hepatic echogenicity with poorer visualization of the intrahepatic vessels and diaphragm. Grade 0 (control) patients had normal liver echogenicity. Grade 1 patients had a minimal diffuse increase in hepatic echogenicity, with normal visualization of the diaphragm and of the walls of the intrahepatic vessel. Grade 2 patients had a moderate diffuse increase in hepatic echogenicity, with slightly impaired visualization of the hepatic veins and diaphragm. Grade 3 patients had a marked increase in echogenicity with poor penetration of the posterior portion of the right lobe of the liver, and poor or no visualization of the hepatic veins and diaphragm. Each subject was asked to stop breathing during shallow inspiration to avoid modifications caused by deep inspiration, and a spectral analysis of the portal venous flow was recorded for at least $5 \mathrm{sec}$ (Scatarige et al. 1984; Karabulut et al. 2004). The point of measurement of the portal vein was the main portal vein proximal to its bifurcation. To characterize the portal vein, the flow peak maximum velocity $\left(\mathrm{V}_{\text {max }}\right)$, peak minimum velocity $\left(\mathrm{V}_{\text {min }}\right)$, mean flow velocity (MFV), and vein pulsatility index (VPI) were obtained in all patients and control subjects.

\section{Statistical analysis}

The statistical analysis was performed using the Statistical Package for the Social Sciences (version 10.0; SPSS, Chicago, IL, USA). The distribution of data was analyzed using the one-sample Kolmogorov-Smirnov test. The data were expressed as the median for numerical variables. The Kruskal-Wallis and Mann Whitney's U-tests were used to compare the medians. Spearman's correlation of rank coefficient was used to assess the relationship between variables. All tests of significance were two-tailed, with $p<0.05$ considered statistically significant.

\section{Results}

Demographic data of the patient were similar 
TABLE 1. Demographics and doppler flow caracteristics of subjects.

\begin{tabular}{lccc}
\hline & $\begin{array}{c}\text { Control group } \\
(n=20)\end{array}$ & $\begin{array}{c}\text { Study group } \\
(n=60)\end{array}$ & $p^{*}$ \\
\hline Age $($ median$)$ & $42.1 \pm 10.93$ & $43.2 \pm 11.97$ & $>0.05$ \\
Sex $(\mathrm{M} / \mathrm{F})$ & $10 / 10$ & $32 / 28$ & $>0.05$ \\
BMI $\left(\mathrm{kg} / \mathrm{m}^{2}\right)$ & $25.2 \pm 1.18$ & $29 \pm 1.41$ & $<0.001$ \\
VPI & $0.31 \pm 1.31$ & $0.21 \pm 2.55$ & $<0.001$ \\
MFV $(\mathrm{cm} / \mathrm{sec})$ & $16.5 \pm 0.67$ & $12.5 \pm 1.89$ & $<0.001$ \\
$\mathrm{~V}_{\text {max }}(\mathrm{cm} / \mathrm{sec})$ & $40.8 \pm 0.92$ & $28.6 \pm 4.37$ & $<0.001$ \\
$\mathrm{~V}_{\min }(\mathrm{cm} / \mathrm{sec})$ & $28.4 \pm 0.59$ & $22 \pm 2.87$ & $<0.001$ \\
\hline
\end{tabular}

*Mann-Whitney's U-test Results are shown as mean \pm S.D.

BMI, body mass index; VPI, pulsatility; MFV, flow velocity; $\mathrm{V}_{\max }$, peak maximum velocity; $\mathrm{V}_{\min }$, peak minimum velocity.

TABLE 2. Demographics and doppler flow caracteristics of patients of subgroups.

\begin{tabular}{|c|c|c|c|c|}
\hline & \multicolumn{3}{|c|}{ Study group $(n=60)$} & \multirow[b]{2}{*}{$p^{*}$} \\
\hline & $\begin{array}{l}\text { Grade } 1 \\
(n=20)\end{array}$ & $\begin{array}{l}\text { Grade } 2 \\
(n=20)\end{array}$ & $\begin{array}{l}\text { Grade } 3 \\
(n=20)\end{array}$ & \\
\hline Age (median) & $38.5 \pm 14.68$ & $48.2 \pm 10.03$ & $43 \pm 8.86$ & $>0.05$ \\
\hline $\operatorname{BMI}\left(\mathrm{kg} / \mathrm{m}^{2}\right)$ & $27.4 \pm 0.56$ & $28.9 \pm 0.74$ & $30.5 \pm 0.60$ & $<0.001$ \\
\hline VPI & $0.27 \pm 1.26$ & $0.20 \pm 1.29$ & $0.17 \pm 1.21$ & $<0.001$ \\
\hline $\mathrm{MFV}(\mathrm{cm} / \mathrm{sec})$ & $14.6 \pm 0.87$ & $12.6 \pm 0.78$ & $10.3 \pm 0.61$ & $<0.001$ \\
\hline $\mathrm{V}_{\max }(\mathrm{cm} / \mathrm{sec})$ & $34 \pm 0.64$ & $28.5 \pm 0.89$ & $23.5 \pm 0.83$ & $<0.001$ \\
\hline $\mathrm{V}_{\min }(\mathrm{cm} / \mathrm{sec})$ & $24.9 \pm 0.46$ & $22.9 \pm 0.58$ & $18.2 \pm 0.53$ & $<0.001$ \\
\hline
\end{tabular}

*Kruskal-Wallis test. Each $p$ value indicates for Grade 1 vs Grade 2, Grade 2 vs Grade 3 and Grade 1 vs Grade 3. Results are shown as mean \pm S.D.

BMI, body mass index; VPI, pulsatility; MFV, flow velocity; $\mathrm{V}_{\max }$, peak maximum velocity; $\mathrm{V}_{\text {min }}$, peak minimum velocity.

and comparable in both groups $(p>0.05)$. BMI was significantly higher, where as the VPI, MFV, $\mathrm{V}_{\max }, \mathrm{V}_{\min }$, and $\mathrm{V}_{\max }-\mathrm{V}_{\text {min }}$ of the portal vein were all significantly lower in the study group than in the control group $(p<0.001)$ (Table 1$)$. The VPI in the study group was 0.20 . The MFV was 16.5 $\mathrm{cm} / \mathrm{sec}$ in the control group and $12.3 \mathrm{~cm} / \mathrm{sec}$ in the study group with fatty liver. Sonographic grade of fatty liver was negatively correlated with VPI $(\mathrm{r}=-0.946, p<0.001), \mathrm{MFV}(\mathrm{r}=-0.951, p<$ $0.001)$, Vmax $(\mathrm{r}=-0.969, p<0.001)$, and Vmin $(\mathrm{r}=-0.967, p<0.001)$ of the portal vein flow. (Table 2, Figs. 1 and 2).

\section{Discussion}

The relative contributions of intrahepatic fat deposition and inflammatory changes to the flow patterns of the hepatic and portal veins are unknown (Dietrich et al. 1998). The main finding of this study is that the VPI, MFV, $\mathrm{V}_{\max }$, and $\mathrm{V}_{\text {min }}$ decrease in patients with fatty liver. The cause of this change may be reduced vascular compliance in the liver due to fatty infiltration.

Portal venous flow is classically described as being continuous in healthy subjects (Needleman et al. 1986). In normal adults, the VPI is 0.48 (Gallix et al. 1997). Dietrich et al. (1998) investi- 


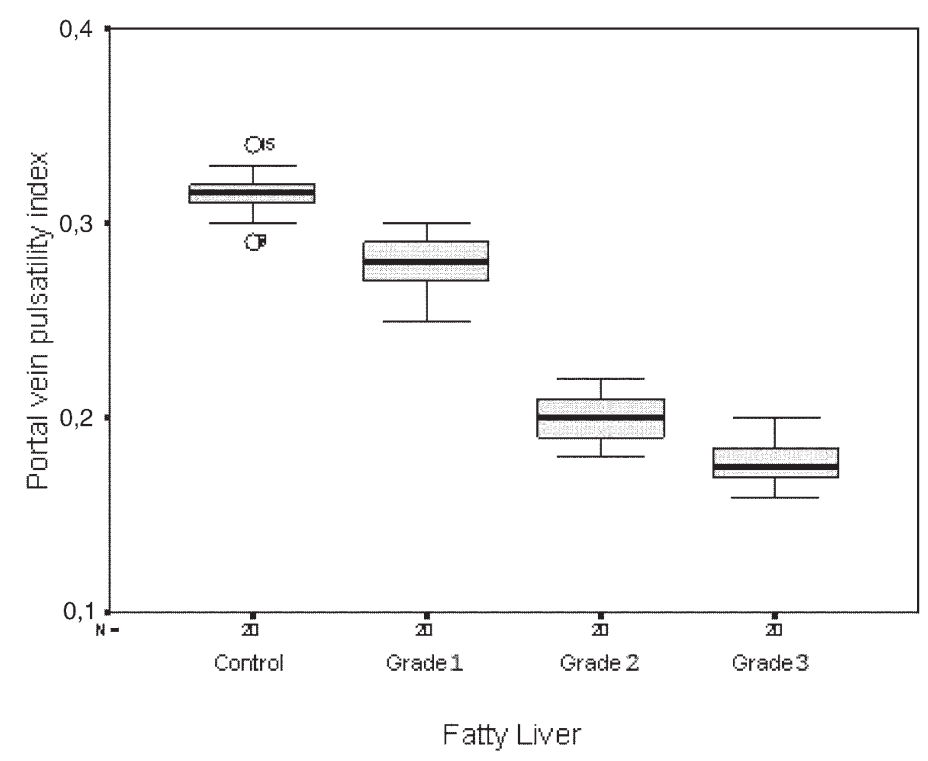

Fig. 1. Portal vein pulsatility. There was a statistically significant negative correlation between the sonographic grade of fatty liver and pulsatility index of portal vein $(\mathrm{r}=-0.946, p<0.001)$.

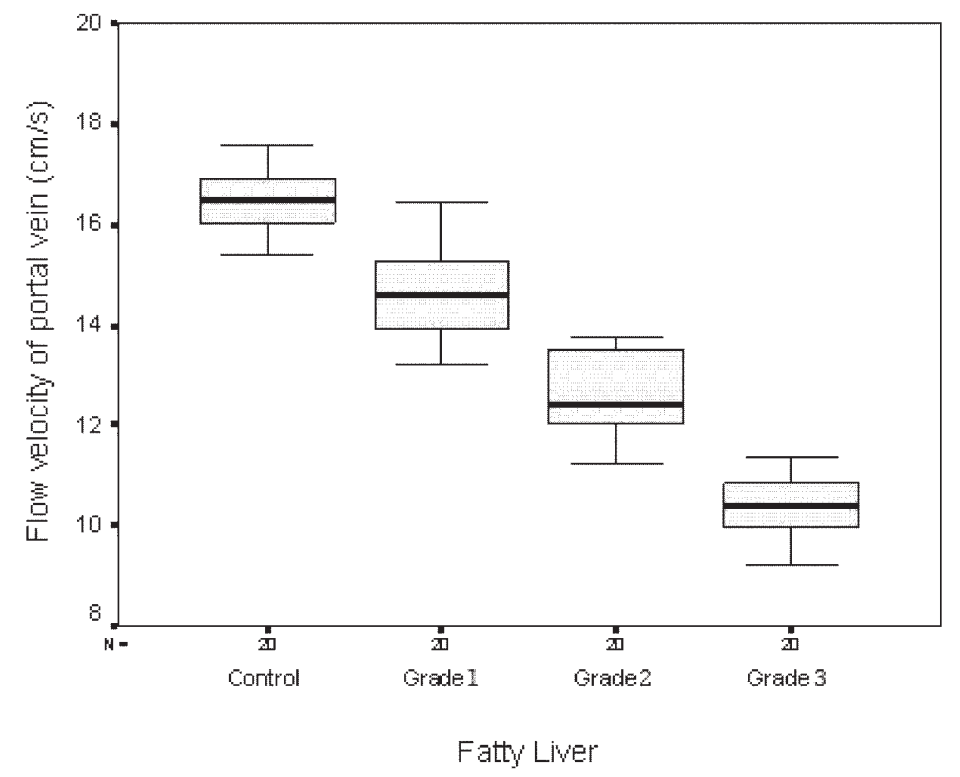

Fig. 2. Flow velocity of portal vein. There was a statistically significant negative correlation between the sonographic grade of fatty liver and flow velocity of portal vein $(r=-0.946, p<0.001)$.

gated the correlation between the $\mathrm{V}_{\max }, \mathrm{V}_{\min }$, and $\mathrm{V}_{\max }-\mathrm{V}_{\text {min }}$ of the portal vein and the degree of fatty infiltration defined histologically in biopsyproven hepatitis- $C$ patients, and found that $\mathrm{V}_{\max }$, $\mathrm{V}_{\min }$, and $\mathrm{V}_{\max }-\mathrm{V}_{\min }$ decreased as the degree of hepatosteatosis increased. Schneider et al. (2005) found a negative correlation between the degree of hepatosteatosis and portal vein flow modulation in patients with hepatitis $\mathrm{C}$, and a negative correlation between the degree of fatty infiltration and the portal vein MFV. The MFV was reported to be decreased in chronic liver diseases also, and it may even reverse in cases with severe fibrosis (Chawla et al. 1998). In our study group the 
patients did not have chronic liver disease, were free of hepatitis B or C, and we also found a negative correlation, between the degree of hepatosteatosis and MFV, VPI, $\mathrm{V}_{\max }$, and $\mathrm{V}_{\min }$ in portal vein flow.

Balci et al. (2008) recently reported a relationship between fatty infiltration of the liver and decreased portal venous velocity and pulsatility in a study of similar design. VPI, MFV, $\mathrm{V}_{\max }$ and $\mathrm{V}_{\min }$ measurements of that study was similar to our findings which supports the idea that portal venous velocity and pulsatility decrease in patients with fatty liver diseases.

There are several limitations to our study: The fatty infiltration was graded based on the ultrasonographic appearance of the liver, since we did not perform liver biopsies in the subjects for ethical reasons. We neither performed echocardiography to exclude heart diseases nor monitor the intra-abdominal pressure during the Doppler examination. The role of the intra-abdominal pressure in the magnitude of venous modulation is evidenced by a decrease in venous pulsatility when the patient is sitting and during deep inspiration, maneuvers known to increase intra-abdominal pressure (Sugerman et al. 1997).

In conclusion, the VPI, MFV, $\mathrm{V}_{\max }$, and $\mathrm{V}_{\text {min }}$ of the portal vein were significantly lower in fatty liver patients and correlated with the grade of hepatosteatosis. The cause of this alteration may be reduced vascular compliance in the liver due to fatty infiltration.

\section{References}

Aube, C., Winkfield, B., Oberti, F., Vuillemin, E., Rousselet, M.C., Caron, C. \& Cales, P. (2004) New Doppler ultrasound signs improve the non-invasive diagnosis of cirrhosis or severe liver fibrosis. Eur. J. Gastroenterol. Hepatol., 16, 743-751.

Balci, A., Karazincir, S., Sumbas, H., Oter, Y., Egilmez, E. \& Inandi, T. (2008) Effects of diffuse fatty infiltration of the liver on portal vein flow hemodynamics. J. Clin. Ultrasound, 36, 134-140.

Bargallo, X., Gilabert, R., Nicolau, C., Garcia-Pagan, J.C.,
Ayuso, J.R. \& Bru, C. (2006) Sonography of Budd-Chiari syndrome. AJR Am. J. Roentgenol., 187, 33-41.

Bayard, M., Holt, J. \& Boroughs, E. (2006) Nonalcoholic fatty liver disease. Am. Fam. Physician,. 73, 1961-1968.

Bolognesi, M., Sacerdoti, D., Mescoli, C., Bombonato, G., Cillo, U., Merenda, R., Giacomelli, L., Merkel, C., Rugge, M. \& Gatta, A. (2007) Different hemodynamic patterns of alcoholic and viral endstage cirrhosis: analysis of explanted liver weight, degree of fibrosis and splanchnic Doppler parameters. Scand. J. Gastroenterol., 42, 256-262.

Chawla, Y., Santa, N., Dhiman, R.K. \& Dilawari, J.B. (1998) Portal hemodynamics by duplex Doppler sonography in different grades of cirrhosis. Dig. Dis. Sci., 43, 354-357.

Dietrich, C.F., Lee, J.H., Gottschalk, R., Herrmann, G., Sarrazin, C., Caspary, W.F. \& Zeuzem, S. (1998) Hepatic and portal vein flow pattern in correlation with intrahepatic fat deposition and liver histology in patients with chronic hepatitis C. AJR Am. J. Roentgenol., 171, 437-443.

Flegal, K.M., Carroll, M.D., Kuczmarski, R.J. \& Johnson, C.L. (1998) Overweight and Obesity in the United States: prevalence and trends, 1960-1994. Int. J. Obes. Relat. Metab. Disord., 22, 39-47.

Gallix, B.P., Taourel, P., Dauzat, M., Bruel, J.M. \& Lafortune, M. (1997) Flow pulsatility in the portal venous system: a study of Doppler sonography in healthy adults. AJR Am.J. Roentgenol., 169, 141-144.

Karabulut, N., Kazil, S., Yagci, B. \& Sabir, N. (2004) Doppler waveform of the hepatic veins in an obese population. Eur. Radiol., 14, 2268-2272.

Kral, J.G., Thung, S.N., Biron, S., Hould, F.S., Lebel, S., Marceau S., Simard, S. \& Marceau, P. (2004) Effects of surgical treatment of the metabolic syndrome on liver fibrosis and cirrhosis. Surgery, 135, 48-58.

Needleman, L., Kurtz, A.B., Rifkin, M.D., Cooper, H.S., Pasto, M.E. \& Goldberg, B.B. (1986) Sonography of diffuse benign liver disease: accuracy of pattern recognition and grading. AJR Am. J. Roentgenol., 146, 1011-1015.

Neuschwander-Tetri, B.A. (2001) Fatty liver and nonalcoholic steatohepatitis. Clin. Cornerstone, 3, 47-57.

Mihmanli, I., Kantarci, F., Yilmaz, M.H., Gurses, B., Selcuk, D., Ogut, G., Altug, A. \& Uysal, O. (2005) Effect of diffuse fatty infiltration of the liver on hepatic artery resistance index. J. Clin. Ultrasound, 33, 95-99.

Salgado, Jr., W., Santos, J.S., Sankarankutty, A.K., Castro, E. \& Silva, O.D. (2006) Nonalcoholic fatty liver disease and obesity. Acta Cir. Bras., 21, 72-78.

Scatarige, J.C., Scott, W.W., Donovan, P.J., Siegelman, S.S. \& Sanders, R.C. (1984) Fatty infiltration of the liver: ultrasonographic and computed tomographic correlation. $J$. Ultrasound Med., 3, 9-14.

Schneider, A.R., Teuber, G., Kriener, S. \& Caspary, W.F. (2005) Noninvasive assessment of liver steatosis, fibrosis and inflammation in chronic hepatitis $\mathrm{C}$ virus infection. Liver Int., 25, 1150-1155.

Sugerman, H., Windsor, A., Bessos, M. \& Wolfe, L. (1997) Intra-abdominal pressure, Sagittal abdominal diameter and obesity comorbidity. J. Intern. Med., 241, 71-79. 